\title{
LA NOCIÓN DE DISCURSO EN HEIDEGGER: UNA ANTICIPACIÓN DE DÉCADAS A LA MODERNA TEORÍA DEL DISCURSO
}

The Notion of Discourse in Heidegger: Anticipation by Decades of the Modern Discourse Theory

EDUARDO RISCO DEL VALLE

Universidad de Los Lagos (Chile)

erisco@ulagos.cl

Resumen

Heidegger practica un abordaje de la noción de discurso que, por la magnitud de su obra y por el carácter más o menos marginal que esta noción tiene en ese conjunto, no ha merecido todavía un tratamiento sistemático. En esta comunicación intentamos recoger algunos elementos relevantes de esa tematización y enfatizamos la relación que ella manifiesta con su temprana concepción de la significación. Concluimos que la tematización del discurso que Heidegger desarrolla se adelanta en décadas a lo que es hoy la teoría contemporánea del discurso.

Palabras clave: Discurso; significación; Heidegger.

Abstract

Heidegger makes an approach to the notion of discourse which, to de magnitude of his work and the more or less marginal character that this notion has in that group, has not yet deserved systematic treatment. In this communication we try to collect some relevant elements of this thematization and emphasize the relationship that it manifests with its early conception of meaning. We concluded that the discourse thematization that Heidegger develops is decades ahead of what contemporary discourse theory is today.

Key words: Discouse; meaning; Heidegger.

\section{INTRODUCCIÓN}

En el marco de la prolífica reflexión heideggeriana acerca del lenguaje, el discurso ocupa un lugar que se manifiesta cada vez más relevante en la medida en que toma cuerpo más definido en lo que se ha dado en llamar el "giro hermenéutico" (Kehre) de su filosofía y cuyo punto de inflexión más relevante puede situarse en su obra de 1927, Ser y Tiempo. Son diversos los autores que han leído la obra de Heidegger desde la clave de la antes mencionada Kehre como un medio capaz de conferirle un nuevo tipo de coherencia; Alvarado (Alvarado, 2004), por ejemplo, sostiene que "antes de este momento [la publicación de Sein und Zeit], aunque el lenguaje había sido liberado de su interpretación exclusivamente lógica (el juicio como lugar de la verdad), Heidegger no tenía ni el método ni los conceptos adecuados para realizar una descripción completa de las determinaciones ontológicas del lenguaje” (p. 84). Carrera (2013), a su vez, subraya el 
hecho de que: "hay un claro cambio de perspectiva entre el análisis existencial del Dasein en Ser y Tiempo y, dentro de este, la consideración acerca del discurso (Rede) como constitutivo ontológico del hombre, y los desarrollos filosóficos posteriores, donde el Habla (Sprache), toma el lugar central en relación a la pregunta por el ser” (p. 12). Mientras que Fontaine de Visscher (Fontaine, 1966) ya había afirmado que la Kehre constituía un: "círculo hermenéutico en el que él [Heidegger] se encontró preso desde el comienzo de su investigación" (p. 225). Lo cierto es que la interrogación heideggeriana acerca del Ser parece indisociable de la pregunta por el Ser y la posición del lenguaje y, en ese marco, de la pregunta por el Ser, las formas y la función del discurso; pregunta a la que Heidegger llega empujado también por sus anteriores reflexiones en torno al lenguaje; las que dan forma a una auténtica filosofía del lenguaje en la que sus disquisiciones acerca del discurso actúan como uno más de los "constructibilia" de su edificio filosófico.

En las líneas que siguen pretendemos poner en relieve la particular concepción de discurso elaborada por Heidegger, su novedad para la época y su anticipación a las nociones de la contemporánea teoría del discurso.

\section{DISEÑO DE LA INVESTIGACIÓN}

Los resultados de la investigación que presentamos en esta comunicación corresponden a una indagación bibliográfica con enfoque hermenéutico que se centró sobre todo en las siguientes obras-fuentes de Heidegger:

- Teoría de las Categorías y de la Significación en Duns Escoto (versión original en alemán como Die Kategorien und Bedeutungslehre des Duns Scotus). (Heidegger, 1916)

- Ser y Tiempo (Heidegger) (versión original en alemán como Sein und Zeit) (Heidegger, 2006).

Incorporándose, en los casos relevantes, la bibliografía crítica a la que hemos tenido acceso tanto en lengua española como alemana y francesa.

En una perspectiva metodológica, hemos procedido a la lectura de los originales en alemán para reconocer ítemes léxicos relevantes (análisis de contenido asistido por ordenador) con selección intencionada y contextualizada de textos atingentes al objeto de indagación (noción de discurso en Heidegger) y la correspondiente revisión de estos elementos en las traducciones españolas a las que hemos podido tener acceso y que se consignan en la bibliografía.

Entre los problemas relevantes que hemos encontrado en este esfuerzo pueden mencionarse los siguientes: en primer lugar, la inexistencia de versiones en español para la primera de las obras-fuente consignadas, por lo que las versiones españolas de la misma 252 | ALPHA № 53 (Diciembre 2021) PÁGS. 251-262. ISSN 07 16-4254 
se consignan con la abreviatura TN (traducción nuestra), salvo para el caso de la excelente versión de las conclusiones de esta obra realizada por Pedro Mantas España (Mantas, 2012); en segundo lugar, el hecho de que las traducciones de Sein und Zeit (en menor medida) y de otros textos del mismo autor (en mayor medida) adolecen de dos dificultades fundamentales: son poco cuidadosas en el tratamiento de los pasajes referidos al discurso e intentan "germanizar" la versión española del conjunto, situación respecto de la cual mostraremos un ejemplo más adelante.

\section{LA INQUIETUD DE HEIDEGGER POR EL LENGUAJE}

La inquietud de Heidegger por el lenguaje es bastante temprana y se remonta ya a su trabajo de 1914 acerca de la concepción del juicio en el psicologismo (Die Lehre vom Urteil im Psycologismus), pero, superando el sesgo logicista que esta obra mantiene, quizá en donde mejor se manifiesta esta preocupación sea en su trabajo de habilitación como académico en 1916 y que versa respecto de la doctrina de las categorías y la significación en Duns Escoto (Die Kategorien und Bedeutungslehre des Duns Scotus), obra en la que, además de criticar el abordaje del tema hecho por la filosofía del lenguaje de su época, llega a trazar un incipiente programa de lo que (él considera) debería ser el objeto y procedimiento de la filosofía del lenguaje. Se trata de una preocupación que cobrará mayor solidez en 1927 con la publicación de Ser y Tiempo y con los trabajos sobre la poesía de Hölderlin (Hörleuterungen zu Hölderlin Dichtung) situados de manera cronológica entre 1936 y 1968 con el notable paréntesis que supone De camino al Habla (Unterwegs zur Sprache) publicado en 1959. Sin embargo, esta inquietud de Heidegger por el lenguaje, más allá de las coordenadas históricas, describe un itinerario de cuatro vectores que, interrelacionados, permiten la captación de este hecho como un fenómeno. Es el primer vector, llama la atención su preocupación (casi obsesiva) por asir las particularidades semánticas de los textos que consulta en su lengua original, preocupación que lo lleva a incluir profusas citas en griego y latín en sus obras sin proporcionar traducción alguna. Es el segundo de estos vectores, su esfuerzo por precisar en todo momento la referencia específica de los conceptos que utiliza y de diferenciarlos (cuando corresponde) de su uso habitual en alemán, lo que lo lleva a la creación de abundantes neologismos basados en las posibilidades aglutinantes del alemán y que han suscitado no pocos quebraderos de cabeza a sus traductores. El tercer vector, por su parte, está constituido por su esfuerzo de fundamentación etimológica de sus acuñaciones lingüísticas que, además de incorporar largos excursos en sus trabajos, en ocasiones resultan de dudosa validez. El cuarto vector, por último, lo constituye la vacilación que se observa (en su mayoría presente en Ser y Tiempo) a la hora de asignar un lugar preeminente sea al Dasein o al lenguaje; cuestión esta última particularmente relevante porque podría haber significado, en el marco de esa obra, una superación de la filosofía del sujeto que Lafont (Lafont, 1993) plantea en los siguientes términos: 
... que tendría que haber arrebatado al Dasein su 'carácter preeminente', abriendo con ello la posibilidad de superar efectivamente el esquema sujeto-objeto de la filosofía de la conciencia, es ignorado sistemáticamente en SyT. Por el contrario, el esquema sujeto-objeto, mantenido implícitamente en la dicotomía Dasein - entes distintos del Dasein, determina desde el principio hasta el final la estructura central de esta obra (p. 188).

\section{DE LA SIGNIFICACIÓN AL DISCURSO}

Aun cuando en el Habilitationschrift Heidegger todavía no entiende el discurso más que como sinónimo de expresión: "el aporte de la lengua mira hacia la completa comunicación del sentido un discurso" (TN) [Die Leistung der Sprache zielt also auf die vollendete Mitteilung des Sinnes einer Rede] (Heidegger, 1978, p. 305), su tratamiento de la teoría de la significación proporciona un elenco suficiente de elementos que operan como bloques constructivos de lo que después será su teoría del discurso. En efecto, su abordaje de la significación opera en tensión con las nociones de conciencia y realidad, por lo que su primera aproximación presenta a la significación como "contenido verbal no sensible" (TN) [unsinnlichen Wortgehalt] (p. 307), con lo que la significación se transforma en "algo" a lo que es posible acceder desde la forma sensible de las palabras [Die Sinnlich wahnehbare Wortgestalt] (p. 307) y cuya importancia reside en que es omnipresente en el pensamiento y el discurso: "El dominio de las significaciones atraviesa de parte a parte, sea cual sea su especie o su perfección, el discurso y la comunicación científica, como también el proceso mismo del pensamiento sistemáticamente ordenado" (TN) [Der Bedeutungsbereich durchzieht, wie geartet und unvollkommen auch immer er sein mag, die wissenschaftliche Rede und Mitteilung ebenso wie den systematisch geordneten wissenschaftichen Gedankenzug] (p. 306).

En Sein und Zeit, la tematización de la significación reaparece en forma explícita en el parágrafo $§ 18$ en un sistema de triple coordenada que vincula los términos significar (Bedeuten), significaciones (Bedeutungen) y significatividad (Bedeutsamkeit) en el marco de las relaciones que las remisiones permiten hacer visibles. Significar consiste en hacer visible, patente o perceptible la red o plexo de relaciones que (mediante remisión) vincula a los entes que comparecen en el mundo entre sí y con el Dasein manifestando una cadena (no siempre lineal) que va desde "un para un algo, este un para-esto, este un término del dejar-ser en respectividad, y este a aquello que está en condición respectiva" (Heidegger, 2017, p. 115) [ein Um-zu, dieses ein Dazu, dieses ein Wobei des Bewendenlassens, dieses ein Womit der Bewandtnis] (Heidegger 2006, p. 87) entendiendo por "respecto" la relación que el ente contrae con otros que comparecen con él en el ahí del Dasein. Sin embargo, significar no solo constituye un medio para hacer visible los respectos, sino también el procedimiento mediante el cual el Dasein se da a 
entender a sí mismo (se explica) su estar en el mundo y que tendrá su función fundamental en el comprender (con-prender). Esta actividad interpretante del Dasein tiene como resultado las significaciones, es decir, manifestaciones localizadas de los respectos a las que Heidegger asigna una particular importancia pues "fundan la posibilidad de la palabra y el lenguaje" (Heidegger 2017, p. 116) [das mögliche Sein von Wort und Sprache fundieren] (Heidegger 2006, p. 187), Por último, el todo respeccional o relacional que se abre mediante el procedimiento del significar (como fenómeno y como actividad interpretativa del Dasein) es lo que Heidegger designa como Significatividad, una abstracción que, en opinión de Lafont (Lafont ,1993), "es entendida como algo distinto del lenguaje y que supuestamente lo fundamenta" (p. 189).

\section{EL DISCURSO EN LA DISQUISICIÓN HEIDEGGERIANA}

La noción de Rede como Discurso es tal vez uno de los aspectos más relevantes de la tematización heideggeriana acerca del lenguaje y probablemente la más trabajada en los escritos posteriores a Sein und Zeit. En esta obra, este tratamiento aparece de manera amplia enriquecido, pero su aprehensión en español topa en forma indeclinable con los problemas de la traducción. En efecto y, en primer lugar, mientras Gaos (Heidegger, 1986) lo traduce en general como "habla", Rivera (Heidegger, 2017) opta por el término "discurso", aunque se permite una licencia en el parágrafo $\S 68$ que complica mucho la captación del sentido heideggeriano del término, ya que traduce el siguiente pasaje: "die Rede faktisch sich zumeist in der Sprache ausspricht" (Heidegger, 2006, p. 349) como: "el discurso se expresa regularmente por medio del lenguaje y el habla" (Heidegger, 1986, p. 367), en donde es claro que traduce "ausspricht" como "habla" y no como "expresado" o "hablado" que es lo que a nuestro juicio correspondería traducir toda vez que en todas las otras ocurrencias del ítem léxico "ausspricht" opta por traducirlo como "expresado"; Gaos, por su parte, traduce este mismo pasaje como: "el habla se expresa de facto ordinariamente en el lenguaje" (p. 337), en donde es posible apreciar el hecho de que prescinde del término "ausspricht", pero introduce la dificultad de identificar discurso con habla e instala, con ello, un texto que parece por completo contradictorio con el de Rivera, como puede apreciarse en la siguiente tabla de contraste:

\begin{tabular}{|c|c|}
\hline \multicolumn{2}{|c|}{ die Rede faktisch sich zumeist in der Sprache ausspricht" } \\
\hline $\begin{array}{c}\text { el discurso se expresa regularmente por } \\
\text { medio del lenguaje y el habla } \\
\text { (Heidegger, 2017, p. 367) Rivera }\end{array}$ & $\begin{array}{c}\text { el habla se expresa de facto ordinariamente en } \\
\text { el lenguaje }\end{array}$ \\
(Heidegger, 1986, p. 337) Gaos \\
\hline
\end{tabular}

En segundo lugar, la opción traductiva seguida por Gaos ha persistido en muchos trabajos ya que aparece justificada por Adrián (Escudero, 2009) en los siguientes términos: "la alternativa 'habla'... permite mantener la misma raíz de las expresiones 'hablar' (Reden), 'hablantes' (Redende), 'hablar de' (in der Reden stehen), 'modo de 
hablar' (Redeweise) y 'habladuría' (Gerede)" (p. 141). Se trata, por supuesto, de un argumento muy atractivo porque simplifica de manera práctica el procedimiento traductivo por vía etimológica, siempre que el objetivo de la traducción sea el "germanizar" la versión española, con lo que la misma ya deja de ser una traducción y se limita a manifestar el fenómeno del contacto entre lenguas en uno de sus efectos más negativos: la interferencia.

Para resolver el problema que suscita este pasaje y debido a que es el único en la traducción Rivera que ofrece esta dificultad, proponemos traducir la expresión "Sprache ausspricht" como "lenguaje expresado" o, mejor aún, como "lenguaje oral", una opción esta segunda que puede resultar conveniente para la interpretación de otros pasajes en obras posteriores. Agregaremos, por último, que al no existir en alemán un ítem léxico que permita diferenciar discurso (Rede) de habla (Rede) se hace preciso mirar en forma cuidadosa el contexto de ocurrencia, y esto es así porque incluso en la traducción alemana del Cours de Linguistique Générale de Saussure a menudo se incorpora el vocablo francés entre paréntesis (parole) para situar en particular la referencia conceptual al habla.

¿Hace Heidegger esta diferencia? A nuestro parecer sí, o por lo menos sí es posible identificar pasajes en los que es claro que se refiere a Rede como algo distinto de Habla (Parole, en términos saussurianos y entendida como el uso individual del código de la lengua) tal como puede apreciarse en el siguiente fragmento (que retomaremos más adelante) "[el discurso] viene a palabra" (Heidegger, 2017, p. 186) [kommt zu Wort] (Heidegger, 2006, p. 161), en donde puede apreciarse que por Rede se refiere a algo distinto del uso individual de la lengua.

Hecha esta precisión, la noción de Rede (discurso) en Sein und Zeit se presenta, como en casi todos los teóricos posteriores del discurso, como un tipo de articulación; pero hay dos notas más relevantes, originales y adelantadas a su época que caracterizan a la noción heideggeriana. Es la primera que lo articulado no es el resultado de un ejercicio cognitivo o de un conjunto de textos dotados de cierta regularidad, sino un cierto tipo de "experiencia" del Dasein en su trato con los entes que comparecen en la circunspección: "El discurso es la articulación de la comprensibilidad" (Heidegger 2017, p. 186) [Rede ist die Artikulation der Verständlichkeit.] (Heidegger, 2006, p.161), es decir, de la aprehensión que el Dasein hace de las relaciones remisionales que vinculan a los entes $a$ la mano que comparecen en la circunspección y que, a su vez, remiten al todo de la significatividad, y de ahí que "A lo articulado en la articulación del discurso lo llamamos el todo de significaciones" (Heidegger, 2017, p.186) [Das in der redenden Artikulation Gegliederte als solches nennen wir das Bedeutungsganze.] (Heidegger, 2006, p. 161) y que este todo articulado pueda "descomponerse en significaciones" (Heidegger, 2017, p. 186) [in Bedeutungen aufgelöst werden] (Heidegger, 2006, p. 161). Recordaremos, a este efecto, que ya en el Habilitationschrift Heidegger había planteado que a la significación 
se accedía por medio de la forma sensible de las palabras [Die Sinnlich wahnehbare Wortgestalt] (Heidegger, 1978, p. 307).

Es la segunda nota original de esta concepción de discurso, que hemos anunciado más arriba, el modo de presentar la relación discurso-lenguaje entendido este último como medio de expresión del primero. En efecto, Heidegger afirma que esta articulación de la comprensibilidad (el discurso) "viene a palabra. A las significaciones les brotan palabras, en vez de ser las palabras las que, entendidas como cosas, se ven provistas de significaciones" (Heidegger 2017, p. 186) [kommt zu Wort. Den Bedeutungen wachsen Worte zu. Nicht aber werden Wörterdinge mit Bedeutungen versehen] (Heidegger, 2006, p. 161). Se trata, por supuesto, de una afirmación muy interesante, entre otras cosas, porque explica muy claro la afirmación más provocativa de los inicios del parágrafo $\S 34$ : "El fundamento ontológico-existencial del lenguaje es el discurso" (Heidegger, 2017, p. 186). [Das existenzial-ontologische Fundament der Sprache ist die Rede] (Heidegger, 2006, p. 160).

En el discurso, sea o no expresado en lenguaje, Heidegger reconoce también una taxonomía compuesta por ocho modos discursivos definidos no por regularidades textuales, sino más bien por intencionalidades comunicativas; estas son "el asentir y disentir, el exhortar y prevenir, en cuanto discusión, consulta e intercesión y también el modo de "hacer declaraciones" (Heidegger, 2017, p. 187) [als zu- und absagen, auffordern, warnen, als Aussprache, Rücksprache, Fürsprache, ferner als "Aussagen machen"] (Heidegger, 2006, p. 161). Se trata, por supuesto, de una taxonomía que recuerda con facilidad la Teoría de los Actos de Habla, pero con la que la teorización heideggeriana de la época de Sein und Zeit no puede estar relacionada más que como antecedente previo a la misma toda vez que la obra de Austin (1962), fundadora de esta teoría (How to do Thinks with Words) es más de cuatro décadas posterior. Lo que sí puede afirmarse es que la taxonomía heideggeriana parece haber sido confeccionada desde una perspectiva en particular interactiva de la comunicación ${ }^{1}$ que, por lo pronto, subraya el carácter/vocación dialógica del discurso mas allá de si este está o no expresado en lenguaje; lo que no podía ser de otra manera toda vez que "El discurso es la articulación significante de la comprensibilidad del estar-en-el-mundo, estar-en-el-mundo al que pertenece el coestar, y que siempre se mantiene en una determinada forma del convivir ocupado" (Heidegger, 2017, p. 187) [Reden ist das "bedeutende" Gliedern der Verständlichkeit des In-der-Welt-seins, dem das Mitsein zugehört, und das sich je in einer bestimmten Weise des besorgenden Miteinanderseins hält] (Heidegger, 2006, p. 161). No

\footnotetext{
${ }^{1}$ Lo que constituye otro rasgo de "adelantamiento" cronológico en la teoría del discurso, pues la aparición de una concepción interactiva opuesta a la concepción tradicional de la comunicación (que se limita al estudio de la transferencia de información entre un emisor activo y un receptor pasivo) deberá esperar todavía a la obra de Katherine Kerbrat-Orecchioni Les Interactions verbales que aparecerá recién en 1990. En esta obra se realiza una explícita comparación entre la teoría tradicional de la comunicación y la perspectiva interactiva de la misma.
} 
obstante lo anterior y aunque el discurso precede a su expresión lingüística, Heidegger reivindica la importancia de esta expresión al señalar que los caracteres existenciales del discurso son "el sobre-qué del discurso (aquello acerca de lo cual se discurre), lo discursivamente dicho en cuanto tal, la comunicación y la notificación" (Heidegger, 2017, p. 187) [das Worïber der Rede (das Beredete), das Geredete als solches, die Mitteilung und die Bekundung] (Heidegger, 2006, p. 165) y agrega más adelante que:

En la forma linguística fáctica de un determinado discurso algunos de estos elementos pueden faltar o bien pasar inadvertidos. El hecho de que frecuentemente no se expresen 'en palabras', no es sino el índice de un modo particular del discurso, ya que el discurso como tal comporta siempre la totalidad de las estructuras mencionadas (Heidegger, 2017, p. 188) [In der faktischen Sprachgestalt einer bestimmten Rede können einzelne dieser Momente fehlen, bzw. unbemerkt bleiben. Daß sie oft "wörtlich" nicht zum Ausdruck kommen, ist nur der Index einer bestimmten Art der Rede, die, sofern sie ist, je in der Ganzheit der genannten Strukturen sein muß] (Heidegger, 2006, p. 163).

Desde nuestra perspectiva de lingüista, por supuesto, no podemos hacer menos que reconocer en estos fragmentos una insinuación incipiente de los dos aspectos que la teorización contemporánea del discurso admite como inseparables: el de la discursividad (su "ontología" en términos heideggerianos) y el de la textualidad (lo que podría ser su carácter "óntico" en el mismo código heideggeriano)2; una diferenciación que Heidegger nunca explicita respecto del discurso, pero que permanece como sustrato y que explica la oscilación hacia el terreno de la textualidad que manifiesta el siguiente fragmento del parágrafo $\S 56$ : "El discurso nos ofrece información acerca de algo y lo hace en un determinado respecto. De aquello a lo que el discurso se refiere de esta manera, él extrae lo que dice, cada vez, en cuanto es este particular discurso, lo dicho en cuanto tal." (Heidegger, 2017, p. 294) [Sie gibt über etwas Aufschluß 3 und das in bestimmter Hinsicht. Aus dem so Beredeten schöpft sie das, was sie je als diese Rede sagt, das Geredete als solches] (Heidegger, 2006, p. 272) que se entiende mejor por recurrencia a un fragmento del parágrafo $§ 35$, en el que Heidegger señala la razón por la que el discurso pasa del modo de existencia del callar al de lo expresado y que sería la comunicación o la necesidad de ella, entendiéndose por su finalidad "llevar al que escucha a una participación en el estar vuelto aperiente hacia lo dicho en el discurso" (Heidegger, 2017, p. 193) [den Hörenden in die Teilnahme am erschlossenen Sein zum Beredeten der Rede zu bringen] (Heidegger, 2006, p. 168).

\footnotetext{
${ }^{2}$ Rivera, en su traducción de Sein und Zeit y en la nota CXXXIX, proporciona sustento argumental a esta afirmación nuestra al señalar que: "El lenguaje es la manifestación óntica (en palabras, signos, gestos) de esa estructura ontológica que es la Rede: es lo que Heidegger expresará más adelante cuando diga que a las significaciones (articuladas en la Rede) "les brotan palabras".
}

258 | AlPHA № 53 (DiCIEMBRE 2021) PÁGS. 251-262. ISSN 07 16-4254 
DISCURSO Y HABLADURÍA (GEREDE): CONSUMO PRODUCTIVO VERSUS CONSUMOPASIVO

$\mathrm{Si}$ el discurso es la experiencia significativa/significante del Dasein de su mundo circundante y esta experiencia puede manifestarse por medio del lenguaje (textos), este discurso puede asumir dos formas, a saber, la de la Rede como tal (entendida como discurso original); y la de la Gerede (habladuría), entendida como una manifestación textual del discurso original que, al estar investida de "materialidad lingüística", se inscribe en el ciclo de la circulación textual y es susceptible de convertirse en insumo de nuevas producciones discursivas (esta vez en el ciclo del "consumo discursivo") hasta el extremo de perder la explicitud de su relación original con el ente del que se habla y transformase en un conjunto de formulaciones lingüísticas que son consumidas y reproducidas en nuevas instancias de generación discursiva, tal como ocurre en la cita enmarcada en la argumentación de autoridad y también en su forma más extrema: la falacia ad baculum. A esta forma del discurso es a la que Heidegger llama Gerede (habladuría en la traducción de Rivera) y que, aunque se cuida de puntualizar que no se trata de una noción peyorativa toda vez que "Terminológicamente significa un fenómeno positivo, que constituye el modo de ser del comprender y de la interpretación del Dasein cotidiano" (Heidegger, 2017, p. 192) [Er bedeutet terminologisch ein positives Phänomen, das die Seinsart des Verstehens und Auslegens des alltäglichen Daseins konstituiert] (Heidegger, 2006, p. 167), lo cierto es que esta nota semántica 'peyorativa' emerge de manera frecuente en el tratamiento de la misma noción como veremos más adelante.

Ya hemos indicado que la Rede es la comprensión que el Dasein alcanza de su entorno fenomenológico y que se expresa en lenguaje (texto), pero:

Esta comprensión que está depositada en la expresión lingüística concierne tanto a la manera, alcanzada o recibida ${ }^{3}$, como se descubre el ente, cuanto a la correspondiente comprensión del ser, y a las posibilidades y horizontes disponibles para una ulterior interpretación y articulación conceptual (Heidegger, 2017, p. 193). [Das so in der Ausgesprochenheit schon hinterlegte Verständnis betrifft sowohl die jeweils erreichte und überkommene Entdecktheit des Seienden als auch das jeweilige Verständnis von Sein und die verfügbaren Möglichkeiten und Horizonte fürneuansetzende Auslegung und begriffliche Artikulation] (Heidegger, 2006, p. 168).

Hemos subrayado en este pasaje la idea de que hay dos maneras de descubrir el ente que comparece en la circunspección del Dasein (y, eventualmente, de llegar a la comprensión): una manera alcanzada y una manera recibida; la primera es la que se opera en la Rede, es originaria y mantiene (cuando se expresa en habla) la explicitud de la relación entre la forma lingüística y el ente "mentado"; la segunda, en cambio, es

\footnotetext{
${ }^{3}$ Cursivas nuestras.
} 
mediada y se adquiere por el contacto con la formulación lingüística de otros discursos (temporalmente previos o concomitantes) en los que la circulación ha obnubilado la relación originaria. A esta segunda manera es a la que Heidegger llama Gerede (Habladuría) y se trataría de una forma en la que:

... puesto que el hablar ha perdido o no ha alcanzado nunca la primaria relación de ser con el ente del que se habla, no se comunica en la forma de la apropiación originaria del ente del que se habla, sino por la vía de una difusión y repetición de lo dicho. Lo hablado en cuanto tal alcanza círculos cada vez más amplios y cobra un carácter autoritativo. La cosa es así porque se la dice. ... la habladuría no se limita a la repetición oral, sino que se propaga en forma escrita como 'escribiduría'. El hablar repetidor no se funda aquí tan solo en un oír decir. Se alimenta también de lo leído a la ligera. La comprensión media del lector no podrá discernir jamás entre lo que ha sido conquistado y alcanzado originariamente y lo meramente repetido. Más aún, la comprensión media no querrá siquiera hacer semejante distinción ni tendrá necesidad de ella, puesto que ya lo ha comprendido todo (Heidegger, 2017, p. 193). [weil das Reden den primären Seinsbezug zum beredeten Seienden verloren bzw. nie gewonnen hat, teilt es sich nicht mit in der Weise der ursprünglichen Zueignung dieses Seienden, sondern auf dem Wege des Weiter- und Nachredens. Das Geredete als solches zieht weitere Kreise und übernimmt autoritativen Charakter. Die Sache ist so, weil man es sagt. ... Und zwar bleibt dieses nicht eingeschränkt auf das lautliche Nachreden, sondern breitet sich aus im Geschriebenen als das »Geschreibe«. Das Nachreden gründet hier nicht so sehr in einem Hörensagen. Es speist sich aus dem Angelesenen. Das durchschnittliche Verständnis des Lesers wird nie entscheiden können, was ursprünglich geschöpft und errungen und was nachgeredet ist. Noch mehr, durchschnittliches Verständnis wird ein solches Unterscheiden gar nicht wollen, seiner nicht bedürfen, weil es ja alles versteht] (Heidegger, 2006, pp. 168-169).

Parece claro, a partir de esta cita, que la Gerede constituye una Rede de segundo orden que, aunque conlleva a la comprensión, se muestra solo como un primer paso para la auténtica comprensión, la que reside en el carácter "alcanzado", "conquistado" y no adquirido de esa misma comprensión. Llegamos así, pues y en definitiva, a la verdadera noción heideggeriana de Gerede, la que se entiende ahora como: "esa repetición y difusión, por cuyo medio la inicial falta de arraigo se acrecienta hasta una total carencia de fundamento" (Heidegger, 2017, p. 193) [dadurch sich das schon anfängliche Fehlen der Bodenständigkeit zur völligen Bodenlosigkeit steigert, konstituiert sich das Gerede] (Heidegger, 2006, p. 168) y que parece representada en forma práctica en el discurso referido.

Ya hemos indicado que el esfuerzo de Heidegger por bloquear la interpretación peyorativa del término Gerede parece contradicha en los pasajes que la describen y, aunque ya estas citas antecedentes podrían fungir como argumento suficiente para 
sustentar tal afirmación, el aspecto que parece más relevante en este sentido es el hecho de que la Gerede obstaculiza la comprensión al extremo de que:

Entonces el silencio manifiesta algo y acalla la habladuría. El silencio, en cuanto modo del discurso, articula de forma tan originaria la comprensibilidad del Dasein, que es precisamente de él de donde proviene la auténtica capacidad de escuchar y el trasparente estar los unos con los otros (Heidegger, 2017, p. 190). [Dann macht Verschwiegenheit offenbar und schlägt das »Gerede« nieder. Verschwiegenheit artikuliert als Modus des Redens die Verständlichkeit des Daseins so ursprünglich, daß ihr das echte Hörenkönnen und durchsichtige Miteinandersein entstammt] (Heidegger, 2006, p. 165).

\section{CONCLUSIONES}

La noción de discurso de Heidegger, que aparece por primera vez en Sein und Zeit puede entenderse como prefigurada en su tematización de la significación ya tempranamente presente en su trabajo de habilitación como académico de 1916 y que hemos identificado como Habilitationsrift.

La relación entre significación y discurso se hace explícita en Sein und Zeit bajo la forma de una articulación de la significatividad, cuyos bloques constructivos básicos son las significaciones, las que emergen de las relaciones remisionales que los entes contraen entre sí y con el Dasein en el ahí de la circunspección.

El discurso (la experiencia significativa y significante del Dasein) puede llegar a expresarse en lenguaje, no siendo esto un requisito en la formulación heideggeriana, pero es justo esta expresión lingüística el único medio a partir del cual es posible acceder al discurso mismo.

La tematización heideggeriana, además y pese a sus frecuentes deslizamientos al ámbito de la textualidad, no concibe al discurso como una entidad estática, sino como algo dinámico que se inscribe en el ciclo de la producción-circulación-consumo discursivos y de esta consideración es que emerge la distinción entre Rede (discurso propiamente tal) y Gerede (discurso reproducido) que se asocia a la distinción entre comprensión alcanzada (Rede) y comprensión adquirida (Gerede).

Pese a lo ignorada que esta elaboración heideggeriana fue en el contexto de postguerra, es indudable que tuvo un fuerte impacto en tematizaciones ulteriores en las que se encuentra la Foucault y las de los modernos teóricos del discurso.

Un estudio más pormenorizado de esta teorización heideggeriana debería contemplar la noción de discurso como uno de los "constructibilia" de lo que por ahora podríamos identificar como una filosofía del lenguaje de cuño heideggeriano. 


\section{OBRAS CITADAS}

Alvarado, Nicolás (2004). Lenguaje, ser y posibilidad en Ser y Tiempo. Universitas Philosophica: 77-98.

Austin, John L. (1962). How to do Thinks with Words. Clarendom Press.

Carrera, Luciana (2019). IX Jornadas de Investigación en Filosofía http://www.memoria. fahce.unlp.edu.ar/trab_eventos/ev.2897/ev.2897.pdf 28 de agosto de 2013. IX Jornadas de Investigación en Filosofia http://www.memoria.fahce.unlp. edu.ar/trab_eventos/ev.2897/ev.2897.pdf. 16 de noviembre de 2019.

Escudero, Jesús Adrian (2009). El lenguaje de Heidegger. Diccionario filosófico 19121917. Herder.

Fontaine-De Vissher, Luce (1966). La pensée du langage chez Heidegger. Revue Philosophique de Louvain: 224-262.

Heidegger, Martin (2017). Ser y Tiempo. Traducción, prólogo y notas de Jorge Eduardo Rivera. Universitaria.

— (2006). Sein und Zeit. Max Niemeyer Verlag.

— (1986). Ser y Tiempo. Traducción de José Gaos. Fondo de Cultura Económica.

— (1916). Die Kategorien und Bedeutungslehre des Duns Scotus. In Klostermann, V. Gesamtausgabe I. Abteilung: Veröffentlichte Sriften 1914-1970. Band 1. (pp. 189-412). Universitätdrukerei H. Stütz AG Würtzburg, 1978.

Lafont, Cristina (1993). El papel del lenguaje en Ser y Tiempo. Isegoría 7: 183-196.

Mantas, Pedro (2012). La "conclusión" de la tesis de habilitación de Heidegger. Traducción de Pedro Mantas España. Causiensia Vol. VII: 451-474. 